\title{
BOOLEAN ALGEBRAS OF PROJECTIONS AND RESOLUTIONS OF THE IDENTITY OF SCALAR-TYPE SPECTRAL OPERATORS
}

\author{
by B. DE PAGTER and W. J. RICKER
}

(Received 29th April 1995)

\begin{abstract}
Let $\mathcal{M}$ be a Bade complete (or $\sigma$-complete) Boolean algebra of projections in a Banach space $X$. This paper is concerned with the following questions: When is $\mathcal{M}$ equal to the resolution of the identity (or the strong operator closure of the resolution of the identity) of some scalar-type spectral operator $T$ (with $\sigma(T) \subseteq \mathbb{R}$ ) in $X$ ? It is shown that if $X$ is separable, then $\mathcal{M}$ always coincides with such a resolution of the identity. For certain restrictions on $\mathcal{M}$ some positive results are established in non-separable spaces $X$. An example is given for which $\mathcal{M}$ is neither a resolution of the identity nor the strong operator closure of a resolution of the identity.
\end{abstract}

1991 Mathematics subject classification: 47B40,47D30.

\section{Introduction}

The most natural analogue in Banach spaces of selfadjoint operators in Hilbert spaces is the class of scalar-type spectral operators introduced by $\mathrm{N}$. Dunford in the 1950's, $[4,5]$. These are (bounded) operators $T$ which have an integral representation of the form $T=\int_{\sigma(T)} \lambda d P(\lambda)$, where $P(\cdot)$ is a projection-valued measure defined on the $\sigma$-algebra $\mathcal{B}(\sigma(T))$ of all Borel subsets of the spectrum $\sigma(T)$, of $T$, and which is $\sigma$ additive for the strong operator topology. The measure $P$, necessarily unique, is called the resolution of the identity of $T$; its range $\{P(E) ; E \in \mathcal{B}(\sigma(T))\}$ is then a ( $\sigma$-complete) Boolean algebra of projections.

What about the converse? It is a classical result that every $\sigma$-complete Boolean algebra of selfadjoint projections in a separable Hilbert space is the resolution of the identity of some selfadjoint operator; see [2, p. 134], for example. By the MackeyWermer theorem $[1$, p. 354] it follows that any $\sigma$-complete Boolean algebra of projections (not necessarily selfadjoint) in a separable Hilbert space is the resolution of the identity of some scalar-type spectral operator with real spectrum.

The aim of this note is to consider the question of which Boolean algebras of projections in Banach spaces are a resolution of the identity of some scalar-type spectral operator with real spectrum? Such operators are also referred to as pseudohermitian, [8]. There are known examples of Banach spaces where this question always has a positive answer, usually due to the "special geometry" of the space. For instance, in addition to separable Hilbert spaces, this is the case for any $\sigma$-complete Boolean algebra of projections in a Grothendieck space with the Dunford-Pettis 
property, [9], or in any hereditarily indecomposable Banach space, [11, Appendix]. The main result of this note (c.f. Proposition 2) states that the same conclusion holds in the (extensive) class of all separable Banach spaces. In the final section we consider some relevant examples and results concerning the case of non-separable spaces.

\section{Preliminaries}

In this section we fix the notation, recall some definitions and concepts and establish some basic results needed in the sequel.

The space of all bounded linear operators from a Banach space $X$ into itself is denoted by $L(X)$; it is equipped with the strong operator topology $\tau_{s}$. A Boolean algebra (briefly, B.a.) of projections $\mathcal{M} \subseteq L(X)$ is called Bade complete (resp. $\sigma$ complete) if, for every family (resp. countable family) of elements $\left\{E_{\alpha}\right\} \subseteq \mathcal{M}$ the supremum $\vee_{\alpha} E_{\alpha}$ and infimum $\wedge_{\alpha} E_{\alpha}$ exist in the partial order (of range inclusion) in $\mathcal{M}$ (i.e. $E \leq F$ iff $E X \subseteq F X)$ and $\left(\wedge_{\alpha} E_{\alpha}\right) X=\cap_{\alpha} E_{\alpha} X$ and $\left(\vee_{\alpha} E_{\alpha}\right) X=\overline{\operatorname{span}}\left(\cup_{\alpha} E_{\alpha} X\right)$, where the bar denotes closure; see [1], for example. It is assumed that the identity operator $I \in \mathcal{M}$.

We recall the following definitions, where $\mathcal{M} \subseteq L(X)$ is always a B.a.

(i) A vector $x \in X$ is called separating for $\mathcal{M}$ if $E=0$ whenever $E \in \mathcal{M}$ satisfies $E x=0$.

(ii) For each $x \in X$, the carrier projection $C_{x}$ (if it exists) is the element of $\mathcal{M}$ given by $C_{\mathrm{x}}=\wedge\{E \in \mathcal{M} ; E x=x\}$.

(iii) $\mathcal{M}$ is called countably decomposable iff every pairwise disjoint family $\left\{E_{\alpha}\right\} \subseteq \mathcal{M}$ (i.e. $E_{\alpha} E_{\beta}=0$ whenever $\alpha \neq \beta$ ) is at most countable.

(iv) A non-zero projection $E \in \mathcal{M}$ is called an atom if, whenever $F \in \mathcal{M}$ satisfies $F \leq E$, then either $F=0$ or $F=E$. We say that $\mathcal{M}$ is atomic if there exists a family $\left\{E_{\alpha}\right\}_{\alpha \in A}$ of atoms in $\mathcal{M}$ such that, whenever $E \in \mathcal{M}$ there is a subset $B \subseteq A$ such that $\Sigma_{\alpha \in B} E_{\alpha}=E$, i.e. $E$ is the $\tau_{s}$-limit of the net of finite partial sums of $\left\{E_{\alpha} ; \alpha \in B\right\}$. If $\mathcal{M}$ is Bade complete, then such a family of atoms is necessarily maximal and pairwise disjoint. If $\left\{E_{\alpha}\right\}_{\alpha \in A}$ is countable we say that $\mathcal{M}$ is countably atomic.

Lemma 1. Let $X$ be a Banach space and $\mathcal{M} \subseteq L(X)$ be a B.a.

(i) If $X$ is separable, then $\mathcal{M}$ is Bade complete iff it is Bade $\sigma$-complete (c.f. [7, XVII Lemma 3.21]).

(ii) If $\mathcal{M}$ is Bade o-complete and has a separating vector, then $\mathcal{M}$ is countably decomposable. In particular, $\mathcal{M}$ is Bade complete.

(iii) If $\mathcal{M}$ is Bade $\sigma$-complete and is countably atomic, then $\mathcal{M}$ is Bade complete. 
Proof. (ii) Let $\left\{E_{\alpha}\right\}$ be a family of disjoint elements of $\mathcal{M}$. Then every series $\sum_{j=1}^{\infty} E_{\dot{\alpha}(j)}$, with $\alpha(j) \neq \alpha(k)$ if $k \neq j$, is $\tau_{s}$-convergent, [7, XVII Lemma 3.4]. Hence, if $x \in X$ is a separating vector, then $Z_{n}=\left\{\alpha ;\left\|E_{\alpha} x\right\|>\frac{1}{n}\right\}$ is a finite set, for every $n \in \mathbb{N}$, and so $Z=\cup_{n=1}^{\infty} Z_{n}$ is countable. Moreover, if $\alpha \notin Z$, then $E_{\alpha} x=0$ and so $E_{\alpha}=0$. This shows that $\left\{E_{\alpha}\right\}$ is countable. Hence, $\mathcal{M}$ is countably decomposable. The Bade completeness of $\mathcal{M}$ follows from [6, IV Lemma 11.5].

(iii) Let $\left\{E_{n} ; n \in \mathbb{N}\right\}$ be a countable family of atoms which generate $\mathcal{M}$; see (iv) in the previous definitions. The map $F \mapsto \Sigma_{n \in F} E_{n}$, for $F \subseteq \mathbb{N}$, is a B.a. isomorphism of $2^{\mathbb{N}}$ onto $\mathcal{M}$ and so $\mathcal{M}$ is complete as an abstract B.a. Since $2^{\mathbb{N}}$ is countably decomposable so is $\mathcal{M}$ and the conclusion follows from part (ii).

Remark 1. Let $\mathcal{M}$ be a Bade complete B.a. with a separating vector. Then every Bade $\sigma$-complete Boolean subalgebra of $\mathcal{M}$ is actually Bade complete. This is clear from Lemma 1(ii) as any separating vector for $\mathcal{M}$ is also separating for the subalgebra.

Lemma 2. Let $\mathcal{M} \subseteq L(X)$ be a Bade complete B.a.

(i) $\mathcal{M}$ is countably decomposable iff $\mathcal{M}$ has a separating vector.

(ii) If $X$ is separable, then $\mathcal{M}$ is necessarily countably decomposable.

Proof. (i) Suppose $\mathcal{M}$ is countably decomposable. Let $\left\{C_{x_{x}}\right\}$ be a maximal, disjoint family of carrier projections from $\mathcal{M}$. By hypothesis it is of the form $\left\{C_{x(n)}\right\}_{n=1}^{\infty}$, where we can suppose that $\|x(n)\|=1$, for all $n \in \mathbb{N}$. Let $x_{o}=\sum_{n=1}^{\infty} 2^{-n} x(n)$. By maximality $\vee_{n=1}^{\infty} C_{x(n)}=I$ and $C_{x_{0}}=I$ from which it follows that $x_{o}$ is a separating vector. The converse is immediate from Lemma 1(ii).

(ii) Let $\left\{E_{\alpha}\right\}_{\alpha \in A}$ be a maximal disjoint system and $\left\{x_{n}\right\}_{n=1}^{\infty}$ be a countable dense set in $X$. Since $I=\Sigma_{\alpha} E_{\alpha}$ we have $x_{n}=\Sigma_{\alpha} E_{\alpha} x_{n}$, for each $n \in \mathbb{N}$, and so there is a countable subset $A_{n} \subseteq A$ such that $E_{\alpha} x_{n}=0$, for all $\alpha \notin A_{n}$. By density of $\left\{x_{n}\right\}_{n=1}^{\infty}$ it follows that $E_{\alpha}=0$ whenever $\alpha \notin \cup_{n=1}^{\infty} A_{n}$.

Remark 2. Lemma 2 shows that a Bade complete B.a. in a separable Banach space always has a separating vector. There are examples of Bade complete B.a.'s in nonseparable spaces which also have separating vectors; see Example 1 in Section 4.

An abstractly $\sigma$-complete (resp. complete) B.a. $\mathcal{A}$ is called countably generated if there is a countable subset $\mathcal{B} \subset \mathcal{A}$ such that the smallest (abstract) $\sigma$-complete (resp. complete) B.a. $\mathcal{F} \subseteq \mathcal{A}$ with $\mathcal{B} \subseteq \mathcal{F}$ is $\mathcal{F}=\mathcal{A}$. An examination of [6, III Lemma 8.4] and its proof, together with the fact that every abstract B.a. is isomorphic to a B.a. of subsets of some set, shows that we may assume $\mathcal{B}$ is a subalgebra of $\mathcal{A}$ (i.e. contains 0 and 1 , finite sups and infs and is closed under complements).

Let $\mathcal{M} \subseteq L(X)$ be a B.a. and $x \in X$. The cyclic space, $\mathcal{M}[x]$, generated by $x$ is the closed subspace $\mathcal{M}[x]=\overline{\operatorname{span}}\{E x ; E \in \mathcal{M}\}$. 
Lemma 3. Let $\mathcal{M} \subseteq L(X)$ be a Bade complete B.a. with a separating vector, say $x_{0}$.

(i) $\mathcal{M}$ is countably generated iff its restriction to $\mathcal{M}\left[x_{0}\right]$ is countably generated.

(ii) If $X$ is separable and $x_{0}$ is cyclic for $\mathcal{M}$, then $\mathcal{M}$ is countably generated.

(iii) If $\mathcal{M}\left[x_{o}\right]$ is separable, then $\mathcal{M}$ is countably generated.

Proof. (i) Let $Y=\mathcal{M}\left[x_{o}\right]$, in which case the family $\mathcal{M}_{Y}=\left\{\left.E\right|_{Y} ; E \in \mathcal{M}\right\}$ of restricted operators is a Bade complete B.a. in $L(Y)$. Then $\Phi: \mathcal{M} \rightarrow \mathcal{M}_{Y}$ given by $\Phi(E)=\left.E\right|_{Y}$ is onto and preserves the B.a. operations. Moreover, $\Phi$ is also injective (using the fact $x_{o}$ is a separating vector) and so is an isomorphism between the abstract B.a.'s $\mathcal{M}$ and $\mathcal{M}_{Y}$.

(ii) Let $\left\{E_{n} x_{0}\right\}_{n=1}^{\infty}$ be a countable dense subset of the separable subset $W=\left\{E x_{o} ; E \in \mathcal{M}\right\}$ of $X$. Let $\mathcal{N} \subseteq L(X)$ be the abstract $\sigma$-complete B.a. generated by $\left\{E_{n}\right\}_{n=1}^{\infty}$. By [7, XVII Lemma 3.4] and the Bade completeness of $\mathcal{M}$ it follows $\mathcal{N}$ is Bade $\sigma$-complete. By Remark $1, \mathcal{N}$ is Bade complete.

Let $E \in \mathcal{M}$. Since $\mathcal{N}_{x_{0}}=\left\{F x_{o} ; F \in \mathcal{N}\right\}$ is a dense subset of $W$ there exist elements $F_{k} \in \mathcal{N}$, for $k \in \mathbb{N}$, with $E x_{o}=\lim _{k \rightarrow \infty} F_{k} x_{o}$. It follows, for every $x=\Sigma_{j=1}^{m} \alpha_{j} R_{j} x_{o}$ with $\alpha_{j} \in \mathbb{C}$ and $R_{j} \in \mathcal{M}$, that $F_{k} x \rightarrow E x$, as $k \rightarrow \infty$. Since the set of all such vectors $x$ is dense in $X$ and $\sup \left\{\left\|F_{k}\right\| ; k \in \mathbb{N}\right\}<\infty$ we conclude that $F_{k} \rightarrow E$ in $L(X)$, as $k \rightarrow \infty$. This shows that $E$ belongs to the $\tau_{s}$-closure of $\mathcal{N}$ in $L(X)$. But, this $\tau_{s}$-closure is precisely $\mathcal{N},[7$, XVII Corollary 3.8]. It follows that $\mathcal{M}=\mathcal{N}$. So, $\mathcal{M}$ is countably generated.

(iii) follows from (i) and (ii) after noting that the restriction of a Bade complete B.a. to a cyclic subspace is again Bade complete; see Lemma 1(ii) and [7, XVII Corollary 3.11$]$.

Remark 3. Lemmas 2 and 3 imply that every Bade complete B.a. in a separable space $X$ is necessarily countably generated. In particular, in a separable space $X$ every Bade $\sigma$-complete Boolean subalgebra of a Bade complete B.a. $\mathcal{M}$, with $\mathcal{M}$ necessarily countably generated, is also countably generated. This property fails in non-separable spaces. For, let $X$ be the non-separable Hilbert space $\ell^{2}([0,1])$ and, for each set $F \in 2^{[0,1]}$, let $P(F) \in L(X)$ denote the operator in $X$ of multiplication by $\chi_{F}$. Then $\mathcal{M}=\left\{P(F) ; F \in 2^{[0,1]}\right\}$ is a Bade complete B.a. (atomic even) which is countably generated. Indeed, let $\mathcal{R} \subseteq \mathcal{M}$ be any abstractly complete $B$.a. containing the countable family $\mathcal{B}$ of all projections of the form $P([a, b))$ and $P((a, b])$ with both $a$ and $b$ being rational numbers from $[0,1]$. Let $x \in(0,1)$ be arbitrary. Then there exists an increasing sequence $\left\{a_{n}\right\}_{n=1}^{\infty} \subseteq(0, x)$ of rational numbers such that $\lim _{n \rightarrow \infty} a_{n}=x$, in which case $[x, 1]=\cap_{n=1}^{\infty}\left(a_{n}, 1\right]$. It follows that $P([x, 1]) \in \mathcal{R}$. Choose a decreasing sequence $\left\{b_{n}\right\}_{n=1}^{\infty} \subseteq(x, 1)$ of rational numbers such that $\lim _{n \rightarrow \infty} b_{n}=x$, in which case $(x, 1]=\cup_{n=1}^{\infty}\left(b_{n}, 1\right]$. It follows that $P((x, 1]) \in \mathcal{R}$. Hence, also $P(\{x\})=P([x, 1] \backslash(x, 1])$ belongs to $\mathcal{R}$. Clearly $0, I \in \mathcal{R}$ (as $\{0\}=\cap_{n=1}^{\infty}\left[0, \frac{1}{n}\right)$ and $\{1\}=\cap_{n=1}^{\infty}\left(1-\frac{1}{n}, 1\right]$ ) and so $P(\{x\}) \in \mathcal{R}$ for every $x \in[0,1]$. By the abstract completeness of $\mathcal{R}$ it follows that $P(F) \in \mathcal{R}$ for every $F \in 2^{[0,1]}$. That is, $\mathcal{R}=\mathcal{M}$ which shows that $\mathcal{M}$ is countably 
generated as an abstractly complete B.a. However, if $\Sigma=\left\{F \in 2^{[0,1]} ; F\right.$ or $F^{c}$ is countable $\}$, then $\mathcal{N}=\{P(F) ; F \in \Sigma)$ is a Bade $\sigma$-complete Boolean subalgebra of $\mathcal{M}$ which is not countably generated. To see this assume that $\mathcal{N}$ is generated by a countable set $\mathcal{E}=\{P(A(n))\}_{n=1}^{\infty}$ with each $A(n) \in \Sigma$. We may suppose that each $A(n)$ is actually countable, otherwise replace it with its complement. Hence, each $A(n)$ is a countable union of singletons, so we may assume that $\mathcal{N}$ is generated by a sequence of singletons $\left\{P\left(\left\{a_{n}\right\}\right)\right\}_{n=1}^{\infty}$. Let $A=\left\{a_{n}\right\}_{n=1}^{\infty}$ and $\Sigma_{A}$ denote the $\sigma$-algebra of all sets $E \subseteq[0,1]$ for which either $E \subseteq A$ or $E^{c} \subseteq A$. Then $P\left(\Sigma_{A}\right)$ is a $\sigma$-complete B.a. with $\mathcal{E} \subseteq P\left(\Sigma_{A}\right)$. Since $P\left(\Sigma_{A}\right)$ is properly contained in $\mathcal{N}$ we have a contradiction. This argument was suggested by $\mathrm{Dr} H$. Vermeer. We also note that $\mathcal{N}$ is not Bade complete; this does not contradict Remark 1 since $\mathcal{M}$ does not have a separating vector.

A fundamental result of $\mathrm{W}$. Bade, [1, Theorem 3.1], states that if $\mathcal{M} \subseteq L(X)$ is a Bade $\sigma$-complete B.a. and $x_{o} \in X$, then there exists an element $x_{o}^{\prime}$ in the dual Banach space $X^{\prime}$ (called a Bade functional for $x_{o}$ ) with the properties that

(i) $\left\langle E x_{o}, x_{0}^{\prime}\right\rangle \geq 0$, for every $E \in \mathcal{M}$, and

(ii) $E x_{o}=0$ whenever $E \in \mathcal{M}$ satisfies $\left\langle E x_{o}, x_{o}^{\prime}\right\rangle=0$.

We end this section with the following

Lemma 4. Let $\mathcal{M} \subseteq L(X)$ be a countably generated, Bade complete B.a. with a separating vector $x_{0}$. Let $x_{0}^{\prime} \in X^{\prime}$ be a Bade functional for $x_{0}$. Define $\mu: \mathcal{M} \rightarrow[0, \infty)$ by

$$
\mu(E)=\left\langle E x_{o}, x_{o}^{\prime}\right\rangle, \quad E \in \mathcal{M}
$$

Then $\mu$ is a (finite) completely additive and separable measure.

Proof. Let $\mathcal{N}$ be a countable algebra from $\mathcal{M}$ which generates $\mathcal{M}$ as an abstract B.a. Let $\mathcal{N}_{\sigma}$ be the abstract $\sigma$-complete B.a. generated by $\mathcal{N}$. Arguing as in the proof of Lemma 3 it follows that $\mathcal{N}_{\sigma}$ is actually Bade complete and coincides with $\mathcal{M}$. Since $\mathcal{M}=\mathcal{N}_{\sigma}$ and $\mathcal{N}_{\sigma}$ is countably generated it follows that $\mu$ is a separable measure. The complete additivity of $\mu$ is a consequence of the Bade completeness of $\mathcal{M}$.

\section{Separable spaces}

Given a Bade complete (or $\sigma$-complete) B.a. $\mathcal{M} \subseteq L(X)$ the aim of this section is to investigate the following questions: When does there exist a psuedohermitian operator $T=\int_{\sigma(T)} \lambda d P(\lambda)$ such that either $\mathcal{M}=\{P(E) ; E \in \mathcal{B}(\sigma(T))\}$ or $\mathcal{M}$ coincides with the $\tau_{s}$ closure of $\{P(E) ; E \in B(\sigma(T))\}$ ?

Recall that a set function $Q: \Sigma \rightarrow L(X)$ satisfying $Q(\Omega)=I$, where $\Sigma$ is a $\sigma$-algebra of subsets of some set $\Omega$, is called a spectral measure if $Q$ is both $\sigma$-additive for the topology $\tau_{\text {s }}$ and multiplicative (i.e. $Q(G \cap H)=Q(G) Q(H)$, for all $G, H \in \Sigma$ ). The connection between spectral measures and B.a.'s is well known, [7, Ch. XVII]. Indeed, the range of a spectral measure is always a Bade $\sigma$-complete B.a. and conversely, every 
Bade $\sigma$-complete B.a. is the range of some spectral measure (e.g. defined on the Baire subsets of its Stone space). Since the $\tau_{s}$-closure of a Bade $\sigma$-complete B.a. is actually a Bade complete B.a. [7, XVII Lemma 3.23], it follows that a Bade $\sigma$-complete B.a. $\mathcal{M} \subseteq L(X)$ is Bade complete iff $\mathcal{M}$ is a $\tau_{s}$-closed subset of $L(X)$.

An operator $T \in L(X)$ is called scalar-type spectral if there exists a spectral measure $Q: \Sigma \rightarrow L(X)$ and a $Q$-integrable function $f: \Omega \rightarrow \mathbb{C}$ such that $T=\int_{\Omega} f d Q$. Here $Q$-integrability of $f$ is meant in the usual sense of integration with respect to a spectral measure, [3], and is equivalent to $f$ being $Q$-essentially bounded, [7, XVIII Theorem 11(c)]. The spectral measure $P: \mathcal{B}(\sigma(T)) \rightarrow L(X)$ given by $P(F)=$ $Q\left(f^{-1}(F)\right)$, for $F \in \mathcal{B}(\sigma(T))$, satisfies $T=\int_{\sigma(T)} \lambda d P(\lambda)$; it is called the resolution of the identity of $T$.

Lemma 6. Let $\mathcal{M} \subseteq L(X)$ be a Bade $\sigma$-complete B.a. which is countably generated, has a separating vector and contains no atoms. Then $\mathcal{M}$ is the resolution of the identity of some pseudohermitian operator.

Proof. Lemma 1(ii) implies $\mathcal{M}$ is Bade complete. Choose a separating vector $x_{o} \in X$ and a Bade functional $x_{o}^{\prime}$ such that $\left\langle x_{o}, x_{o}^{\prime}\right\rangle=1$. Lemma 4 implies the measure $\mu: \mathcal{M} \rightarrow[0, \infty)$ defined by (1), which takes its values in $[0,1]$ because of our choice of $x_{o}^{\prime}$, is separable, non-atomic and satisfies $\mu(I)=1$. By a result of Carathéodory, [12, p. 321], there is a B.a. isomorphism $\Phi$ of $\mathcal{M}$ onto the measure algebra $\mathcal{A}$ generated by Lebesgue measure in $[0,1]$, which preserves countable sups and infs. If $\mathcal{N}$ denotes the Lebesgue null sets of $\mathcal{B}([0,1])$, then $\mathcal{A}$ is $\mathrm{B}$.a. isomorphic to $\mathcal{B}([0,1]) / \mathcal{N}$. Let $\rho: \mathcal{B}([0,1]) \rightarrow \mathcal{B}([0,1]) / \mathcal{N}$ be the quotient map. Then $P: \mathcal{B}([0,1]) \rightarrow L(X)$ given by $P(G)=\Phi^{-1}(\rho(G))$, for $G \in \mathcal{B}([0,1])$, is a spectral measure whose range is precisely $\mathcal{M}$. Hence, $T=\int_{[0,1]} \lambda d P(\lambda)$ is pseudohermitian and has the desired property.

Given a B.a. $\mathcal{M} \subseteq L(X)$ and $E \in \mathcal{M}$ we define $E \mathcal{M}=\{E F ; F \in \mathcal{M}\}$. Then $E \mathcal{M}$ is interpreted as a B.a. acting in the Banach space $E X$; it is clearly Bade complete (resp. $\sigma$-complete) whenever $\mathcal{M}$ is Bade complete (resp. $\sigma$-complete).

Lemma 7. Let $\mathcal{M} \subseteq L(X)$ be a Bade complete B.a. which is countably generated. Then, for each $E \in \mathcal{M}$, the Bade complete B.a. $E \mathcal{M} \subseteq L(E X)$ is also countably generated.

Proof. Let $\left\{F_{n}\right\}$ generate $\mathcal{M}$ as an abstract B.a. Then $\left\{E F_{n}\right\}$ generates $E \mathcal{M}$ as an abstract B.a. Indeed, let $\mathcal{N} \subseteq L(E X)$ be the complete abstract B.a. generated by $\left\{E F_{n}\right\}$. Since $E \mathcal{M}$ is Bade complete we have $\mathcal{N} \subseteq E \mathcal{M}$. Moreover, if $\mathcal{A}=\{F \in \mathcal{M} ; E F \in \mathcal{N}\}$, then $\mathcal{A}$ is a complete abstract B.a. containing $\left\{F_{n}\right\}$ from which it follows that $\mathcal{A}=\mathcal{M}$. Then $\mathcal{N}=E \mathcal{M}$, as required.

We come to the main result of this section.

Proposition 1. Let $\mathcal{M} \subseteq L(X)$ be a Bade complete B.a. with a separating vector. Then 
$\mathcal{M}$ is the resolution of the identity of a pseudohermitian operator if and only if it is countably generated.

Proof. Suppose that $\mathcal{M}$ is countably generated. By Bade completeness, if $\left\{Q_{\alpha}\right\}$ is a maximal disjoint family of atoms in $\mathcal{M}$, then the series $\Sigma_{\alpha} Q_{\alpha}$ is $\tau_{s}$-summable to the element $Q=\vee_{\alpha} Q_{\alpha}$ of $\mathcal{M}$. Since $\mathcal{M}$ has a separating vector, say $x_{o}$, and $\Sigma_{\alpha} Q_{\alpha} x_{o}=Q x_{o}$ we conclude that $\mathcal{M}$ has at most countably many atoms, say $\left\{Q_{n}\right\}_{n=1}^{\infty}$. Let $Q=\vee_{n=1}^{\infty} Q_{n}$ and $X_{a}=Q X$. Denote the closed subspace $(I-Q) X$ by $X_{c}$, in which case $X=X_{a} \oplus X_{c}$.

Let $\mathcal{M}_{c}=(I-Q) \mathcal{M}$ denote the restriction of $\mathcal{M}$ to $X_{c}$. Then $\mathcal{M}_{c}$ is a Bade complete B.a. in $L\left(X_{c}\right)$ which is countably generated (c.f. Lemma 7), has no atoms and has $(I-Q) x_{o}$ as a separating vector. By Lemma 6 there is a pseudohermitian operator $T_{c} \in L\left(X_{c}\right)$ having $\mathcal{M}_{c}$ as its resolution of the identity.

Now $\mathcal{M}_{a}=Q \mathcal{M}$, the restriction of $\mathcal{M}$ to $X_{a}$, is a Bade complete, countably atomic B.a. in $L\left(X_{a}\right)$. Let $[a, b] \subseteq \mathbb{R}$ be an interval containing $\sigma\left(T_{c}\right)$ in its interior. Then $T_{a}=\sum_{n=1}^{\infty}\left(b+\frac{1}{n}\right) Q_{n}^{(a)}$, where $Q_{n}^{(a)}$ is the restriction of $Q_{n}$ to $X_{a}$ and the series is $\tau_{s}-$ convergent in $L\left(X_{a}\right)$, is a pseudohermitian operator in $X_{a}$ whose resolution of the identity is $\mathcal{M}_{a}$.

Let $P_{c}: \mathcal{B}\left(\sigma\left(T_{c}\right)\right) \rightarrow L\left(X_{c}\right)$ and $P_{a}: \mathcal{B}\left(\sigma\left(T_{a}\right)\right) \rightarrow L\left(X_{a}\right)$ be the resolutions of the identity of $T_{c}$ and $T_{a}$, respectively. By construction $\Lambda=\sigma\left(T_{a}\right) \cup \sigma\left(T_{c}\right)$ is a disjoint union (in $\mathbb{R}$ ). Moreover, every Borel set $G \in \mathcal{B}(\Lambda)$ has a unique decomposition $G=G_{a} \cup G_{c}$ into disjoint Borel sets $G_{a} \in \mathcal{B}\left(\sigma\left(T_{a}\right)\right)$ and $G_{c} \in \mathcal{B}\left(\sigma\left(T_{c}\right)\right.$ ). Define $P(G) \in L(X)$ by $P(G)=P_{a}\left(G_{a}\right) \oplus P_{c}\left(G_{c}\right)$. Then the set function $P: \mathcal{B}(\Lambda) \rightarrow L(X)$ so defined is a spectral measure. Moreover, if $T=T_{a} \oplus T_{c}$, in which case $\sigma(T)=\Lambda$, then $T$ is pseudohermitian with resolution of the identity precisely $P$ and $\mathcal{M}=\{P(G) ; G \in \mathcal{B}(\sigma(T))\}$.

Conversely, suppose $\mathcal{M}=P(\mathcal{B}(\sigma(T)))$ where $P: \mathcal{B}(\sigma(T)) \rightarrow L(X)$ is the resolution of the identity of a pseudohermitian operator $T$. Then $P$ induces a B.a. isomorphism between $\mathcal{M}$ and $\mathcal{B}(\sigma(T)) / \mathcal{N}(P)$ where $\mathcal{N}(P)=\{E \in \mathcal{B}(\sigma(T)): P(E)=0\}$. Let $x_{o}^{\prime}$ be a Bade functional for $x_{0}$ and define $v: \mathcal{B}(\sigma(T)) \rightarrow[0, \infty)$ by $v(E)=\left\langle P(E) x_{0}, x_{0}^{\prime}\right\rangle$, for each $E \in \mathcal{B}(\sigma(T))$. Then $\mathcal{M}$ is B.a. isomorphic with $\mathcal{B}(\sigma(T)) / \mathcal{N}(v)$, where $\mathcal{N}(v)=$ $\{E \in \mathcal{B}(\sigma(T)) ; v(E)=0\}=\mathcal{N}(P)$, and hence $\mathcal{M}$ is countably generated as an abstract complete B.a.

Combining Proposition 1 with Remarks 2 and 3 gives the following result which answers completely the two questions posed at the beginning of the section.

Proposition 2. Let $X$ be a separable Banach space and $\mathcal{M} \subseteq L(X)$ be a Bade $\sigma$ complete B.a. Then $\mathcal{M}$ is the resolution of the identity of a pseudohermitian operator.

\section{Non-separable spaces}

We continue our investigation of the two questions posed at the beginning of Section 3, but now without the requirement that $X$ be separable. 
Let $P: \Sigma \rightarrow L(X)$ be a spectral measure. Then it has associated with it a locally convex Hausdorff space $L^{1}(P)$ of $P$-integrable functions. Moreover, $L^{1}(P)$ is a locally convex algebra (for pointwise operations) and the integration map $I_{P}: L^{1}(P) \rightarrow L(X)$ given by $I_{P}(f)=\int_{\Omega} f d P$ is a bicontinuous isomorphism of $L^{1}(P)$ onto its range $I_{P}\left(L^{1}(P)\right) \subseteq L(X)$. All of these notions and statements can be found in [3], for example. If the underlying $\sigma$-algebra $\Sigma$ is countably generated (as a $\sigma$-algebra), then $L^{1}(P)$ is necessarily separable, [10, Proposition 2], and hence its isomorphic image $I_{P}\left(L^{1}(P)\right)=$ $\left\{\int_{\Omega} f d P ; f \in L^{1}(P)\right\}$ is a $\tau_{s}$-separable subspace of $L(X)$.

Let $T=\int_{\sigma(T)} \lambda d P(\lambda)$ be a pseudohermitian operator in $X$. By the above remarks $I_{P}\left(L^{1}(P)\right)$ is a separable subspace of $L(X)$ and hence, so is its $\tau_{s}$-closure $\overline{I_{P}\left(L^{1}(P)\right)_{s}}$ in $L(X)$. Let $\mathcal{M}$ be the Bade complete B.a. given by taking the $\tau_{s}$-closure of $P(\mathcal{B}(\sigma(T)))$ in $L(X)$. Since $\langle\mathcal{M}\rangle_{s}=\overline{I_{P}\left(L^{1}(P)\right)_{s}}=\langle P(\mathcal{B}(\sigma(T)))\rangle_{s}$, where $\langle\mathcal{M}\rangle_{s}$, denotes the strong operator closed algebra generated by $\mathcal{M}$ in $L(X)$, we have established the following:

Proposition 3. If $\mathcal{M} \subseteq L(X)$ is the $\tau_{3}$-closure of the resolution of the identity of a pseudohermitian operator, then $\langle\mathcal{M}\rangle_{3}$ is necessarily a separable subspace of $L(X)$.

Example 1. Let $(\Omega, \Sigma, \mu)$ be a finite, positive measure space such that $\mu$ is a nonseparable measure, and $X$ denote the non-separable Hilbert space $L^{2}(\mu)$. For each $E \in \Sigma$, let $P(E) \in L(X)$ be the operator in $L^{2}(\mu)$ of multiplication by $\chi_{E}$. Then $\mathcal{M}=P(\Sigma)$ is a Bade $\sigma$-complete B.a. The constant function 1 is a cyclic vector for $\mathcal{M}$, that is, the cyclic space $\mathcal{M}[\mathbf{1}]=X$. In particular, 1 is a separating vector for $\mathcal{M}$ and so $\mathcal{M}$ is actually Bade complete (c.f. Lemma 1 ).

Suppose $T=\int_{\sigma(T)} \lambda d P(\lambda)$ is pseudohermitian and $P(\mathcal{B}(\sigma(T)))=\mathcal{M}$. The map $S \mapsto S 1$ from $\langle\mathcal{M}\rangle_{s}$ into $X$ is continuous and its range $Y$ is dense in $X$ (as 1 is a cyclic vector). Since $\langle\mathcal{M}\rangle$, is $\tau_{s}$-separable (c.f. Proposition 3) it follows $Y$ is separable in $X$ and hence $X=\bar{Y}$ is separable. This contradiction shows no such operator $T$ can exist.

Suppose there was a resolution of the identity $\mathcal{N}$, say, such that $\mathcal{M}$ is the $\tau_{s}$-closure of $\mathcal{N}$. Since $\langle\mathcal{N}\rangle_{s}=\langle\mathcal{M}\rangle_{s}$ it would follow that $\langle\mathcal{M}\rangle_{\text {s }}$ is separable which is already shown not to be the case. Hence, no such $\mathcal{N}$ can exist.

Example 1 shows one cannot have a positive answer to our questions in all nonseparable spaces; some restrictions on $\mathcal{M}$ are needed. For certain classes of atomic B.a.'s something positive can be said, even without a separating vector (c.f. Proposition 1).

Proposition 4. Let $\mathcal{M} \subseteq L(X)$ be a Bade complete B.a. which is atomic and is countably generated as an abstract B.a. Then $\mathcal{M}$ is the strong operator closure of the resolution of the identity of a pseudohermitian operator.

Proof. Let $\left\{E_{n}\right\}$ be a countable algebra generating $\mathcal{M}$ as an abstract B.a. and $\left\{P_{\alpha}\right\}_{\alpha \in A}$ be a maximal family of atoms generating $\mathcal{M}$. For $E \in \mathcal{M}$, let $A(E)=\left\{\alpha \in A ; P_{\alpha} E \neq 0\right\}$ and, for $\alpha \in A$, let $\Phi(\alpha)=\left\{n \in \mathbb{N} ; P_{\alpha} E_{n} \neq 0\right\}$. Denoting $2^{\mathrm{N}}$ by $\triangle$ we have a map $\Phi: A \rightarrow \triangle$. Then $A\left(E_{n}\right)=\Phi^{-1}(\{G \in \Delta ; n \in G\})$ and $E_{n}=\vee_{\alpha \in A\left(E_{n}\right)} P_{a}$, for $n \in \mathbb{N}$. 
Claim. $\Phi$ is injective.

Proof of Claim. Define $\Psi: 2^{\Delta} \rightarrow 2^{\wedge}$ by $\Psi(U)=\Phi^{-1}(U)$, for $U \subseteq \Delta$. Then $\Psi\left(2^{\Delta}\right)$ is a complete B.a. in $2^{A}$ containing the algebra $B=\left\{A\left(E_{n}\right)\right\}_{n=1}^{\infty}$. Since the identification $E \leftrightarrow A(E)$ between $\mathcal{M}$ and $2^{\mathcal{A}}$ is a B.a. isomorphism we can identify the algebra $\left\{E_{n}\right\}_{n=1}^{\infty}$ with $B$ and conclude that $B$ is a countable algebra generating $2^{A}$ as an abstract B.a. So, $\Psi\left(2^{\Delta}\right)$ is a complete B.a. in $2^{A}$ containing $B$ and with $B$ generating $2^{A}$, which implies $\Psi\left(2^{\Delta}\right)=2^{\wedge}$.

Suppose $\alpha_{1} \neq \alpha_{2}$ in $A$. Let $W_{j} \subseteq \Delta$ satisfy $\left\{\alpha_{j}\right\}=\Phi^{-1}\left(W_{j}\right)$, for $j \in\{1,2\}$. If $\Phi\left(\alpha_{1}\right)=\Phi\left(\alpha_{2}\right)=w$ say, then $w \in W_{1} \cap W_{2}$ which implies that both $\alpha_{1}$ and $\alpha_{2}$ belong to $\Phi^{-1}\left(W_{1} \cap W_{2}\right)=\left\{\alpha_{1}\right\} \cap\left\{\alpha_{2}\right\}=\phi$. This is impossible and the claim is established.

The above Claim shows that $\#(A) \leq \#(\Delta)=c$, where \# denotes cardinality. So, we may assume that $A \subseteq[0,1]$. Let $\Lambda=\bar{A}$. Define a spectral measure $P: \mathcal{B}(\Lambda) \rightarrow L(X)$ by $P(F)=\Sigma_{\alpha \in A \cap F} P_{\alpha}$, for each $F \in \mathcal{B}(\Lambda)$. Then $T=\int_{\Lambda} \lambda d P(\lambda)$ is a pseudohermitian operator with $\sigma(T)=\Lambda$ and resolution of the identity $P$. If $E \in \mathcal{M}$, then $E=\vee_{\alpha \in A(E)} P_{\alpha}$ with each $P_{\alpha} \in P(B(\sigma(T)))$. By the Bade completeness of $\mathcal{M}$ it follows that $E$ is the $\tau_{s}$-limit of the net of finite sums $\left\{\Sigma_{\alpha \in F} P_{\alpha}\right\}_{F}$ as $F$ varies through the finite subsets of $A(E)$. This shows that $\mathcal{M}=\overline{P(\mathcal{B}(\sigma(T))) \text { s }}$ and completes the proof of the proposition.

Proposition 4 shows that the countably generated B.a. $\mathcal{M}$ of Remark 3 is the strong operator closure of the resolution of the identity of some pseudohermitian operator. A natural question is whether it is possible to remove the phrase "strong operator closure" from the previous sentence. The following result shows that an answer to this question is dependent on the Continuum Hypothesis.

Proposition 5. Let $\mathcal{M} \subseteq L(X)$ be a Bade complete B.a. which is the resolution of the identity of a pseudohermitian operator. Assuming the Continuum Hypothesis it follows that $\mathcal{M}$ is countably decomposable, and hence has a separating vector.

Proof. Let $\left\{Q_{a}\right\}_{a \in A}$ be any maximal disjoint system in $\mathcal{M}$. For each $F \subseteq A$, let $Q(F)=\vee_{\alpha \in F} Q_{\alpha}$ in which case $F_{1} \neq F_{2}$ iff $Q\left(F_{1}\right) \neq Q\left(F_{2}\right)$. Let $P: \mathcal{B}(\sigma(T)) \rightarrow L(X)$ be the resolution of the identity of a pseudohermitian operator $T$ with $P(B(\sigma(T)))=\mathcal{M}$. For $F \subseteq A$, choose $\hat{F} \in \mathcal{B}(\sigma(T))$ such that $P(\hat{F})=Q(F)$ and $\hat{F}_{1} \neq \hat{F}_{2}$ whenever $F_{1} \neq F_{2}$. Then the map $F \mapsto \hat{F}$ from $2^{A}$ into $\mathcal{B}(\sigma(T))$ is injective and so $\#\left(2^{A}\right) \leq \#(\mathcal{B}(\sigma(T)))=2^{\aleph_{0}}$. Granted the Continuum Hypothesis we deduce $A$ is countable.

Remark 4. For the B.a. $\mathcal{M}$ of Remark 3 it is clear that there is no separating vector. So, granted the Continuum Hypothesis, Proposition 5 implies that $\mathcal{M}$ is not the resolution of the identity of any pseudohermitian operator.

We conclude with an example of a Bade complete B.a. which is countably generated, has no atoms and no separating vector, but is the strong operator closure of the resolution of the identity of a pseudohermitian operator. 
Example 2. Let $\Omega=[0,1) \times[0,1)$. For each $F \subseteq \Omega$, define

$$
F_{t}=\{x \in[0,1) ;(t, x) \in F\}, \quad t \in[0,1) .
$$

Let $\mathcal{B}$ denote the Borel subsets of $[0,1)$ and $\Sigma$ be the $\sigma$-algebra given by

$$
\Sigma=\left\{F \subseteq \Omega ; F_{t} \in \mathcal{B} \text { for all } t \in[0,1\}\right.
$$

Define $\mu: \Sigma \rightarrow[0, \infty]$ by $\mu(F)=\Sigma_{0 \leq t \leq 1} m\left(F_{t}\right)$, for $F \in \Sigma$, where $m: \mathcal{B} \rightarrow[0,1]$ is Lebesgue measure. Then $\mu$ is a non-separable, localizable measure and $\Sigma / \mathcal{N}(\mu)$ is a complete abstract B.a. where $\mathcal{N}(\mu)=\{F \in \Sigma ; \mu(F)=0\}$. Note that $F \in \mathcal{N}(\mu)$ iff $m\left(F_{t}\right)=0$ for all $t \in[0,1)$.

Let $X$ be the non-separable Banach space $L^{\prime}(\mu)$ and $\mathcal{M} \subseteq L(X)$ be the B.a. of all projections $P(E)$, for $E \in \Sigma$, of multiplication in $L^{1}(\mu)$ by $\chi_{E}$. Then $\mathcal{M}$ is Bade complete, has no atoms and no separating vector.

We need some notation. Whenever $J \subseteq \Omega$ is a square, of the form $[a, a+2 u) \times[b, b+2 u)$ say, define a partition $\left\{J_{j}\right\}_{j=0}^{3}$ of $J$ into the 4 smaller squares $J_{o}=[a, a+u) \times[b, b+u)$, $J_{1}=[a+u, a+2 u) \times[b, b+u), J_{2}=[a, a+u) \times[b+u, b+2 u)$ and $J_{3}=[a+u, a+2 u) \times$ $[b+u, b+2 u)$. Now consider the case of $J=\Omega$. For $\ell \geq 1$, define inductively the sets

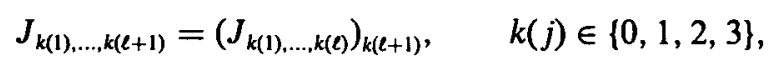

and the functions

$$
f_{\ell}=\sum_{k(1), \ldots, k(\ell)}\left[\frac{k(1)}{4}+\cdots+\frac{k(\ell)}{4^{\ell}}\right] \chi_{k(1), \ldots, k(\ell)}, \quad k(j) \in\{0,1,2,3\},
$$

where $\chi_{k(1), \ldots, k(\ell)}$ is the characteristic function of $J_{k(1) \ldots, k(\ell)}$. Then $\left\{f_{\ell}\right\}_{\ell=1}^{\infty}$ is an increasing sequence of functions with values in $[0,1]$ satisfying $\left\|f_{\ell+1}-f_{\ell}\right\|_{\infty} \leq 3 / 4^{\ell+1}$. If $f$ denotes the pointwise limit of $\left\{f_{\ell}\right\}_{l=1}^{\infty}$ on $\Omega$, then $0 \leq f \leq 1$ and $f$ is $\Sigma$-measurable. Moreover, we have the following properties.

(i) For each integer $\ell \geq 1$,

$$
\frac{k(1)}{4}+\cdots+\frac{k(\ell)}{4^{\ell}} \leq f(w)<\frac{k(1)}{4}+\cdots+\frac{k(\ell)}{4^{\ell}}+\frac{1}{4^{\ell}}, \quad w \in J_{k(1), \ldots, k(\ell)} .
$$

(ii) $f^{-1}\left(A_{k(1), \ldots, k(\ell)}\right)=J_{k(1), \ldots, k(\ell)}$, for each $\ell \geq 1$, where

$$
A_{k(1), \ldots, k(\ell)}=\left\{t \in[0,1) ; \frac{k(1)}{4}+\cdots+\frac{k(\ell)}{4^{\ell}} \leq t<\frac{k(1)}{4}+\cdots+\frac{k(\ell)}{4^{\ell}}+\frac{1}{4^{\ell}}\right\} .
$$

(iii) $f$ is injective and $f(\Omega)$ is dense in $[0,1]$. 
Let $T \in L(X)$ be the pseudohermitian operator in $L^{1}(\mu)$ of multiplication by $f$. Then the family $\mathcal{R}$ of projections $P\left(J_{k(1), \ldots, k(\ell)}\right)$, for all $\ell \geq 1$ and $k(j) \in\{0,1,2,3\}$, belongs to the resolution of the identity of $T$ and hence $\mathcal{M}$ is the strong operator closure of the resolution of the identity of a pseudohermitian operator. Finally, the density of the dyadic rationals in $[0,1]$ can be used to show that the countable family $\mathcal{R}$ generates $\mathcal{M} \simeq \Sigma / \mathcal{N}(\mu)$ as an abstract B.a., that is, $\mathcal{M}$ is countably generated.

\section{REFERENCES}

1. W. G. BADE, On Boolean algebras of projections and algebras of operators, Trans. Amer. Math Soc. 80 (1955), 345-359.

2. J. DixmIER, Von Neumann algebras (North Holland Series, No. 27, Amsterdam, 1981).

3. P. G. DodDS and W. J. RICKER, Spectral measures and the Bade reflexivity theorem, $J$. Funct. Anal. 61 (1985), 136-163.

4. N. Dunford, Spectral theory, II. Resolutions of the identity, Pacific J. Math. 2 (1952), 559-614.

5. N. Dunford, Spectral operators, Pacific J. Math. 4 (1954), 321-354.

6. N. DUNFORD and J. T. SchWARTZ, Linear operators I, 2nd Printing (Wiley-Interscience, New York, 1964).

7. N. DUNFORD and J. T. SCHWARTZ, Linear operators III: spectral operators, (WileyInterscience, New York, 1972).

8. S. Kantorovitz, On the characterization of spectral operators, Trans. Amer. Math. Soc. 111 (1964), 152-181.

9. W. J. RICKER, Spectral operators of scalar-type in Grothendieck spaces with the Dunford-Pettis property, Bull. London Math. Soc. 17 (1985), 268-270.

10. W. J. Ricker, Separability of the $L^{1}$-space of a vector measure, Glasgow Math. J. 34 (1992), 1-9.

11. W. J. Ricker, Well bounded operators of type (B) in H.I. spaces, Acta Sci. Math. (Szeged) 59 (1994), 475-488.

12. H. L. Royden, Real analysis, 2nd Edition (Macmillan Co., London, 1970).

Faculty of Technical Mathematics \& Informatics Department of Pure Mathematics

DelfT University of TechNology

MeKelWEg 4 2628CD DELFT

THE NeTHERLANDS
School of Mathematics UNIVERSITY OF NeW SOUTH Wales SYDNEY, NSW, 2052 Australia 